\title{
Uncovering the genealogy of GW170817 with self- consistent and detailed binary models
}

\author{
Heloise Stevance ( $\nabla$ hfstevance@gmail.com ) \\ University of Auckland https://orcid.org/0000-0002-0504-4323 \\ JJ Eldridge \\ University of Auckland https://orcid.org/0000-0002-1722-6343 \\ Elizabeth Stanway \\ University of Warwick https://orcid.org/0000-0002-8770-809X \\ Joe Lyman \\ University of Warwick https://orcid.org/0000-0002-3464-0642 \\ Anna McLeod \\ Durham University \\ Andrew Levan \\ Radboud University
}

\section{Article}

Keywords:

Posted Date: February 1st, 2022

DOI: https://doi.org/10.21203/rs.3.rs-1297088/v1

License: (c) (i) This work is licensed under a Creative Commons Attribution 4.0 International License.

Read Full License 


\title{
Uncovering the genealogy of GW170817 with self-consistent and detailed binary models
}

\author{
Heloise F. Stevance ${ }^{1, *}$, Jan J. Eldridge ${ }^{1}$, Elizabeth R. Stanway ${ }^{2,}$, Joe Lyman ${ }^{2}$, Anna F. \\ McLeod $^{3,4}$, and Andrew J. Levan ${ }^{2,5}$,
}

\author{
${ }^{1}$ The Department of Physics, The University of Auckland, Private Bag 92019, Auckland, New Zealand \\ ${ }^{2}$ Department of Physics, University of Warwick, Coventry, CV4 7AL, UK \\ ${ }^{3}$ Centre for Extragalactic Astronomy, Department of Physics, Durham University, South Road, Durham DH1 3LE, UK \\ ${ }^{4}$ Institute for Computational Cosmology, Department of Physics, University of Durham, South Road, Durham DH1 \\ 3LE, UK \\ ${ }^{5}$ Department of Astrophysics/IMAPP, Radboud University, PO Box 9010, 6500 GL, The Netherlands \\ *hfstevance@gmail.com
}

\begin{abstract}
Binary neutron star mergers are one the ultimate events of massive binary star evolution, and are a key observable to test several unknowns in modern astrophysics. Our understanding of their parent system is in its infancy and primarily based on studies of the handful of known galactic binary neutron stars. Here we present the first systematic search for progenitors of a specific merger event (GW170817) and recover their full evolution from birth to death. We find with >99\% confidence that GW170817 originated from a $\sim 8_{-5}^{+2}$ Gyr old half-solar metallicity stellar population. Contrary to evolutionary channels previously suggested to explain Galactic binary neutron stars, we find that our most likely progenitor systems undergo common envelope evolution during the life of the primary and that of the secondary. Future observations will be key to untangle the diversity of binary neutron star genealogy, as well as test the accuracy of different evolutionary frameworks.
\end{abstract}

\section{Introduction}

Binary Neutron Star (BNS) mergers have three telltale observational signatures: the Gravitational Waves (GW) released during their in-spiral, the gamma-ray bursts powered by the short-lived central engine resulting from the merger, and the kilonova arising from thermal emission from the neutron-rich ejecta. The first, and so far only, BNS merger for which three signatures were detected was GW170817/GRB170817A/AT2017 $\mathrm{gfo}^{1-8}$. Located $40 \mathrm{Mpc}$ away in the lenticular galaxy NGC 4993, ${ }^{9,10}$, it was the subject of unprecedented follow-up across the entire electromagnetic spectrum, and presented the first opportunity to study the host environment of a confirmed BNS merger. The properties of NGC 4993 were inferred by comparing observed Spectral Energy Distributions (SEDs) to template spectra ${ }^{11-14}$, but widely used models at the time did not account for the evolution of binary systems. Furthermore, while past work has generally demonstrated that the progenitor was likely old (>1Gyr), a systematic approach to the study of the host properties and their implications for the specific progenitors of GW170817 has not yet been considered. In this work we present a new analysis of NGC 4993 using the Binary Population And Spectral Synthesis (BPASS) code. These simulations combine detailed stellar evolution, population synthesis and spectral synthesis to provide a large set of predictions that have been shown to self-consistently reproduce key observables ${ }^{15,16}$. Compared to models which are created and run with a specific analysis in mind, they allow us to perform self-consistent studies without fine-tuning ${ }^{17-23}$. Additional numerical methods now account for supernova kicks and merger time calculations to match our models to the observed properties of compact mergers. We first update the age and metallicity estimate of NG4993, before using these properties to perform the first systematic search for the progenitors of GW170817.

\section{Results}

\section{Metallicity distribution and star formation history of NGC 4993}

We fit our spectral templates (see Method section) to previously published MUSE data obtained on 2017 August 18 at the Very Large Telescope (PI: A. Levan) ${ }^{12}$ to retrieve the age and metallicity distribution of NGC 4993. The majority of the stellar population is found to have a sub-solar metallicity, and roughly $20 \%$ of the light originates from solar and super-solar populations $(Z=0.014,0.020,0.030)$ - in particular, the galactic center shows significant chemical enrichment relative to the outskirts of the galaxy (see Figure 1). The light fraction distribution reveals two main stellar populations: an older component $\geq 3$ Gyrs with a peak $\sim 8$ Gyrs ago which constitutes the majority of the lower metallicity ( $\mathrm{Z}=0.010$ ) population and a younger 

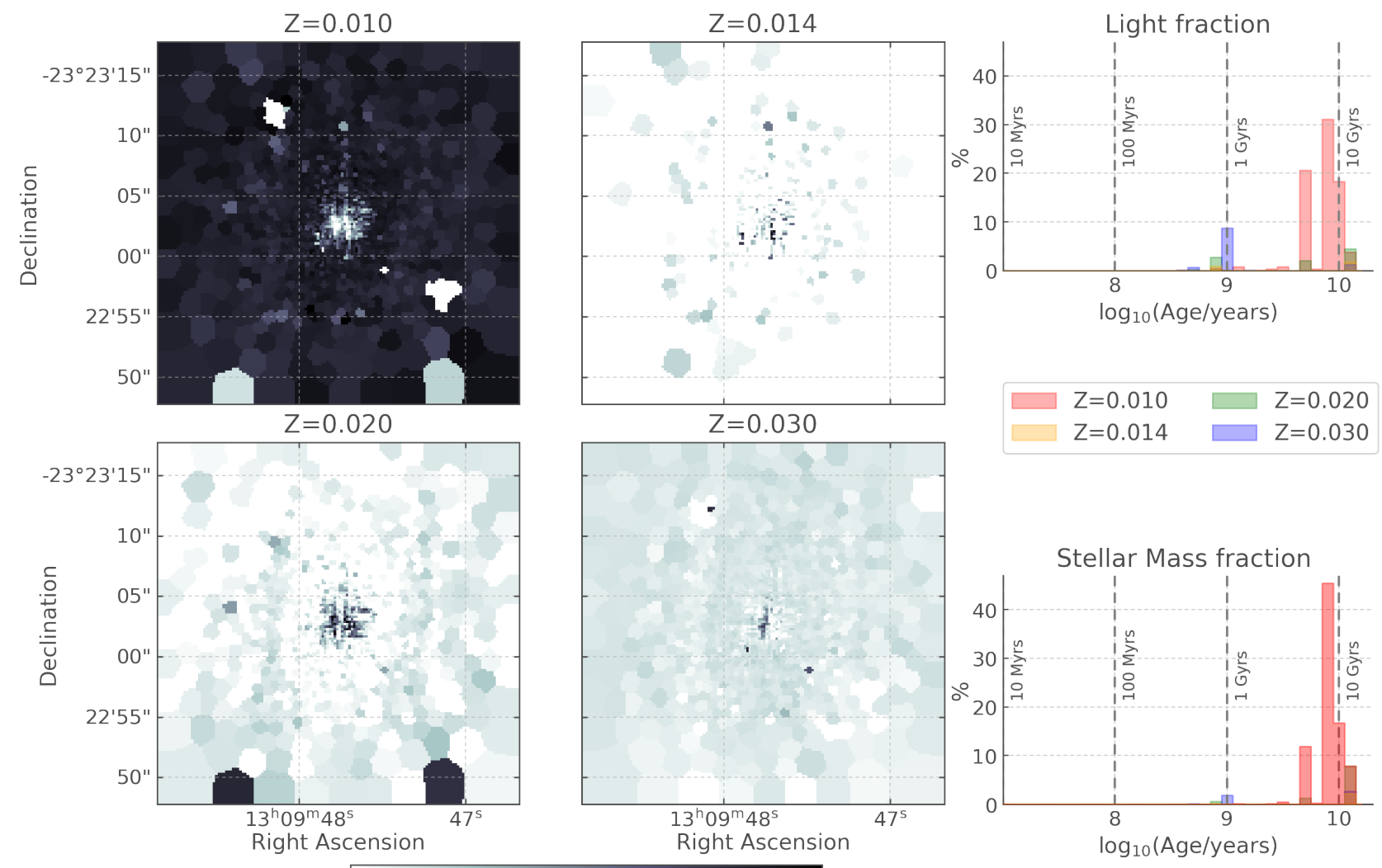

Figure 1. The left and middle panels present metallicity maps of NGC 4993 as inferred by fitting BPASS templates to the observed spectra in each bin. The locations of AT $2017 \mathrm{gfo}$ and a foreground star are masked (most visible in the top left panel) as the Voronoi bins associated with these sources are subject to light contamination resulting in poor fitting of the underlying galactic population. They are not included in the age-metallicity dependent mass fraction and light fraction plots shown in the right-most panels. 

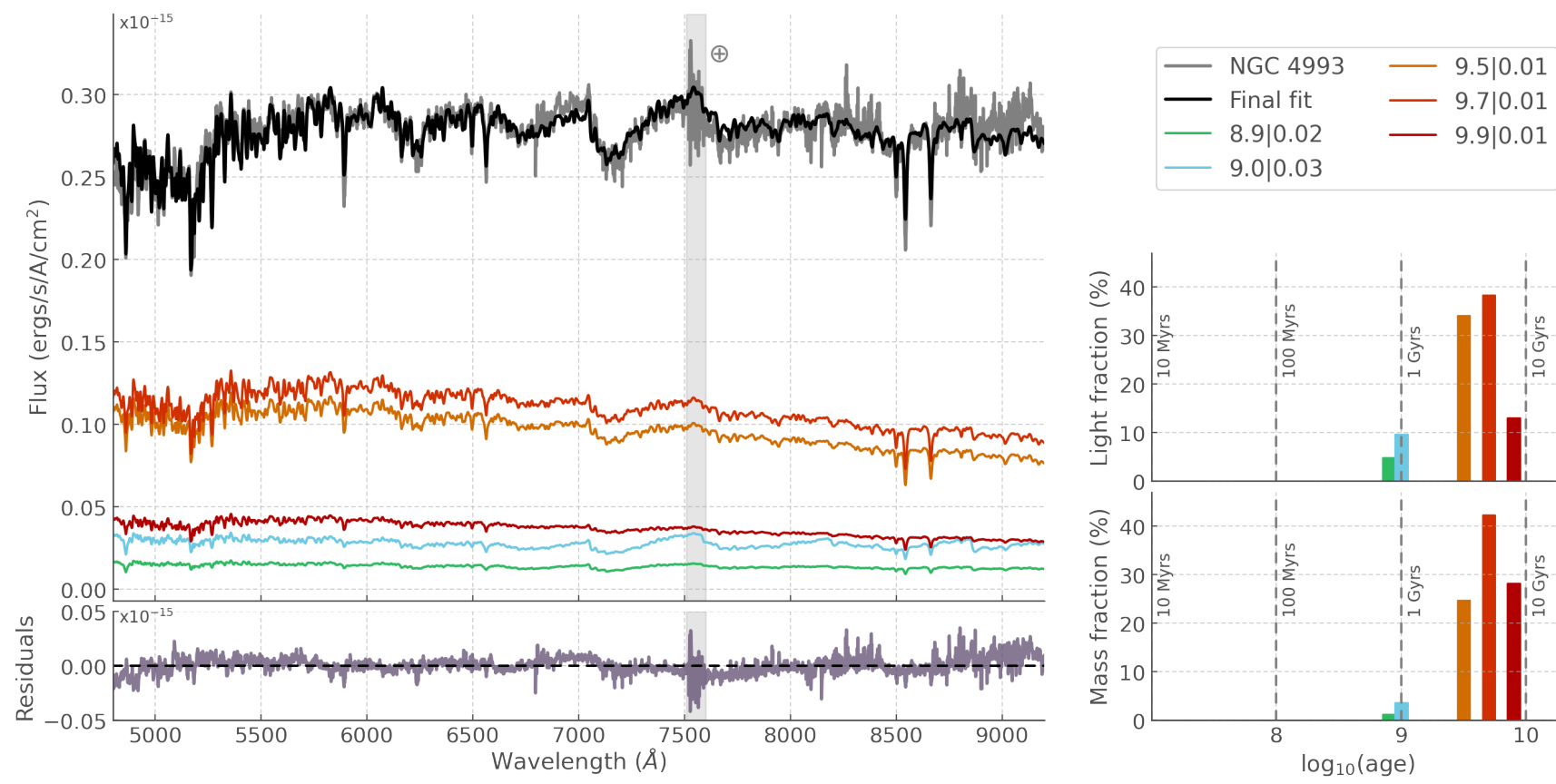

Figure 2. Best fit to the SED of the population in an annulus around the location of AT2017gfo and corresponding age and metallicity distribution. The coloured SEDs in the top left panel are the individual spectral components summed to obtain the final fit - the log age and metallicity are provided in the legend. The grey shaded area highlights a spectral region contaminated by telluric features that cannot be easily removed.

Table 1. Summary of the search criteria and corresponding results. The "Unique systems" are pairs of primary and secondary star evolutionary pathways; the "total matches" is the number of unique solutions retrieved from our simulations which sampled the kick distribution 10,000 times for each supernova - each Unique System can result in many matches if a large range of kicks allow for a GW170817-like merger to occur. The rates of GW170817-like events within each population is also shown, as well as the probability $\mathrm{P}_{Z}$ that a given population hosted GW170817.

\begin{tabular}{|c|c|c|c|c|c|}
\hline \multicolumn{2}{|c|}{ Search criteria } & \multicolumn{4}{|l|}{ Results } \\
\hline Metallicity & Delay time range (Gyrs) & Total matches & Unique systems & Rate $\left(\mathrm{M}_{\odot}^{-1} \mathrm{yr}^{-1}\right)$ & $\mathrm{P}_{Z}$ \\
\hline 0.010 & $2.5-10$ & 134,555 & 4,234 & $6.2 \times 10^{-17}$ & 0.991 \\
\hline 0.020 & $0.8-1.3$ & 53,061 & 4,842 & $3.6 \times 10^{-16}$ & 0.004 \\
\hline 0.030 & $0.8-1.3$ & 24,064 & 3,283 & $1.3 \times 10^{-16}$ & 0.005 \\
\hline
\end{tabular}

(0.8 to $1.3 \mathrm{Gyr}$ ) population with solar/super-solar metallicity $(\mathrm{Z}=0.020,0.030)$ - see Figure 1 . We also recover the SED of the stellar population at the location of the event (see Methods) and we find that the results (Figure 2) are in line with the pattern observed in the galaxy at large, with the older population representing $~ 85 \%$ (95\%) of the light (mass) fraction.

\section{Genealogy of GW170817}

We now search for BNS merger events with delay times matching the inferred, metallicity-dependent, ages. We also ensure that the chirp-mass of candidate progenitors is within $\pm 0.1 \mathrm{M}_{\odot}$ of the chirp-mass derived for GW170817 $\left(1.186 \mathrm{M}_{\odot}{ }^{24}\right)-$ this is 10 times larger than the reported uncertainty, which reflects the fact that our models have a discrete range of initial properties and cannot be expected to match observations to two decimal places. The search criteria and results are shown in Table 1 .

We recover over 200,000 matches, where a match is a combination of a unique primary evolution pathway, a unique secondary evolution pathway, and final supernova kick value (this particular number therefore is dependent on the number of kicks applied, see Method section). Not all systems are equally likely to occur in nature: The occurrence rate $\left(N_{\text {imf }}\right)$ of individual stellar models is dependent on the initial mass function, binary fraction and period distribution; we must also take into account the fraction of supernova kicks that result in a delay time consistent with GW170817 ( $\left.f_{\text {kicks }}\right)$. To retrieve the probability that a population with a given metallicity will host a GW170817-type event, we can normalise across all metallicities 

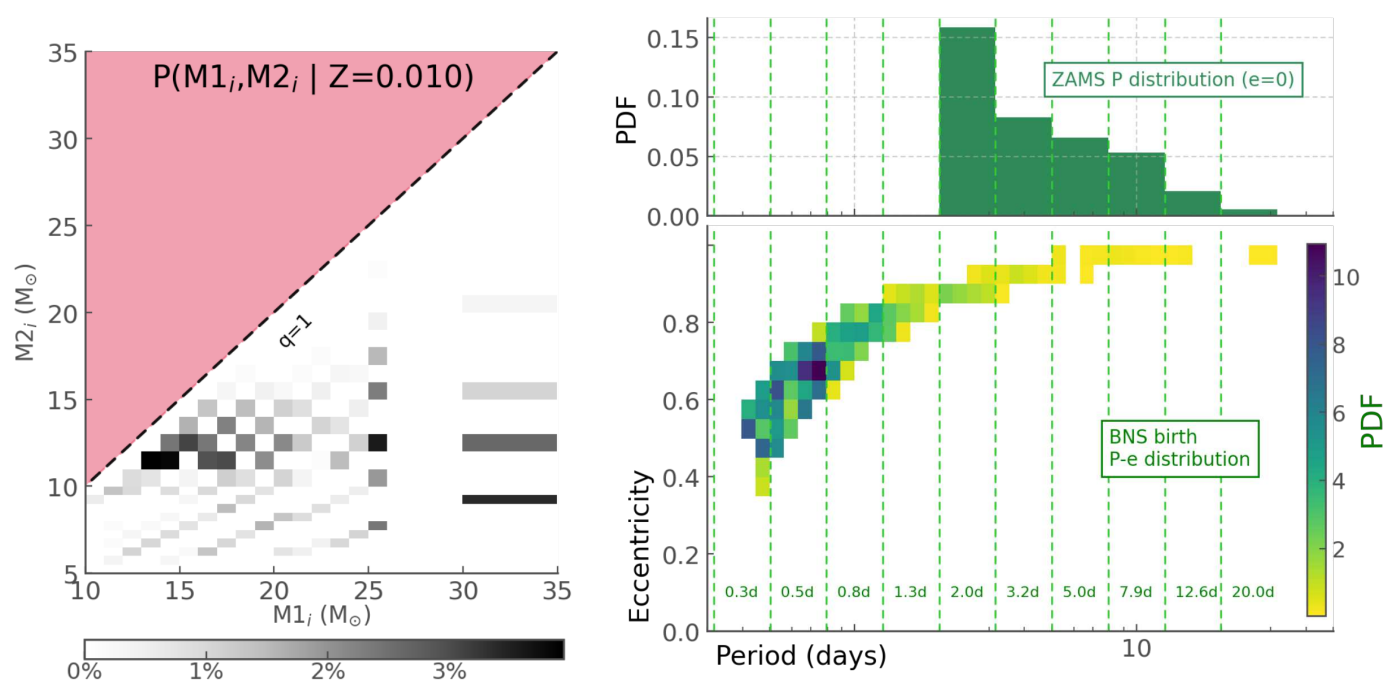

Figure 3. Left panel: Initial mass distribution across the best $68 \%$ of models predicting GW170817-like events. The red region shows the unphysical region where $\mathrm{q}>0$. Right panel: Initial $(\log )$ orbital period Probability Distribution Function (PDF) for our $68 \%$ best systems (top) and Period-eccentricity diagram of their BNS at birth (bottom). Note that the initial periods are taken from a grid of $\log (\mathrm{P} /$ days $)$ starting at 0 with 0.2 dex increments. The final periods are not bound by this grid but were plotted on the same log scale and binned to 0.05 dex bins.

and multiply by the relevant mass fraction $\left(f_{\text {mass }}\right)$ :

$$
P_{Z}=\frac{\sum_{i}\left(N_{\text {imf }}\right)_{i, Z} \cdot\left(f_{\text {kick }}\right)_{i, Z}}{\sum_{i, Z}\left(N_{\text {imf }}\right)_{i, Z} \cdot\left(f_{\text {kick }}\right)_{i, Z}} \times f_{\text {mass }, Z} .
$$

In doing so, we find that there is a $>99 \%$ chance that GW170817 originated from the old $\mathrm{Z}=0.010$ population, even though the rate of GW170817-like events per $\mathrm{M}_{\odot}$ is $\sim 2$ to 6 times greater in the younger populations (as expected from previous studies $^{25}$ ). Of the $>4000$ matching systems (unique combination of primary and secondary star evolution regardless of kick value) in the $\mathrm{Z}=0.010$ population, the first 399 contribute $68 \%$ of the probability - we focus on these most likely models for the rest of the analysis. All the percentages provided henceforth have been weighted by the occurrence rate $N_{\text {imf }}$ such that they reflect what we expect to see in nature.

The Zero-Age Main-Sequence (ZAMS) mass distribution (see Figure 3) reveals two main types of progenitors: lower mass systems with primary $\mathrm{M}_{1, \text { ZAMS }} \sim 13-20 \mathrm{M}_{\odot}$ and a mass ratio $\left(\mathrm{q}:=M_{2} / M_{2}\right) \gtrsim 0.55$, and higher mass systems with primary $\mathrm{M}_{1, \text { ZAMS }} \sim 25-30 \mathrm{M}_{\odot}$ and $\mathrm{q} \lesssim 0.55$. The initial orbital periods of the binary system are very short (see Figure 3), with $94 \%$ having $\mathrm{P}_{Z A M S}<16$ days. We find that $>90 \%$ of primary stars undergo a hydrogen shell-burning (case B) Common Envelope (CE) phase with $11 \%$ of those being preceded by stable Roche-Lobe Overflow (RLOF) during the main-sequence (MS), also called Case A RLOF. The systems undergoing this evolution all have $\mathrm{M}_{1, Z A M S} \gtrsim 20 \mathrm{M}_{\odot}$ and low $\mathrm{q}(\sim 0.34)$. Additionally, roughly $9.5 \%$ of primaries do not undergo a CE, instead they exhibit Case A RLOF as a result of their very short initial orbital period (2.5 days). After the CE phase (or MS RLOF) has ended, all primary stars are stripped to a surface hydrogen mass fraction (X) of $<0.32$, but only $26 \%$ are sufficiently stripped to be He stars. By the end of their life, however, $52 \%$ are $\mathrm{H}$ free $(X<0.001)$ and all primaries have $\mathrm{X}<0.25$ and surface hydrogen mass $<0.012 \mathrm{M}_{\odot}$ resulting in hydrogen deficient $(\mathrm{Ib} / \mathrm{c})$ supernovae $e^{26,27}$. Consequently, significant stripping has occurred between the end of the CE phase and the supernova. This is achieved through two main mechanisms: wind mass-loss and helium-shell burning (Case BB) mass transfer. We find that 30\% of primaries undergo Case BB RLOF, but in most cases (82\%) the wind mass loss is the dominant stripping mechanism in the late stages of evolution.

There is less variety in the evolutionary pathways of the secondary stars: We find that in $100 \%$ of our best progenitor candidates, the secondary star undergoes Case B CE. Furthermore, 78\% will initiate Case BB RLOF, but for $>50 \%$ of them the mass loss from strong stellar winds still dominates. Finally, the BNS at birth show preferred periods $<1$ day - the few models with initial BNS period $\gtrsim 5$ days require very high initial eccentricities $>0.95$ to merge with the right delay time (see Figure 3 ). 


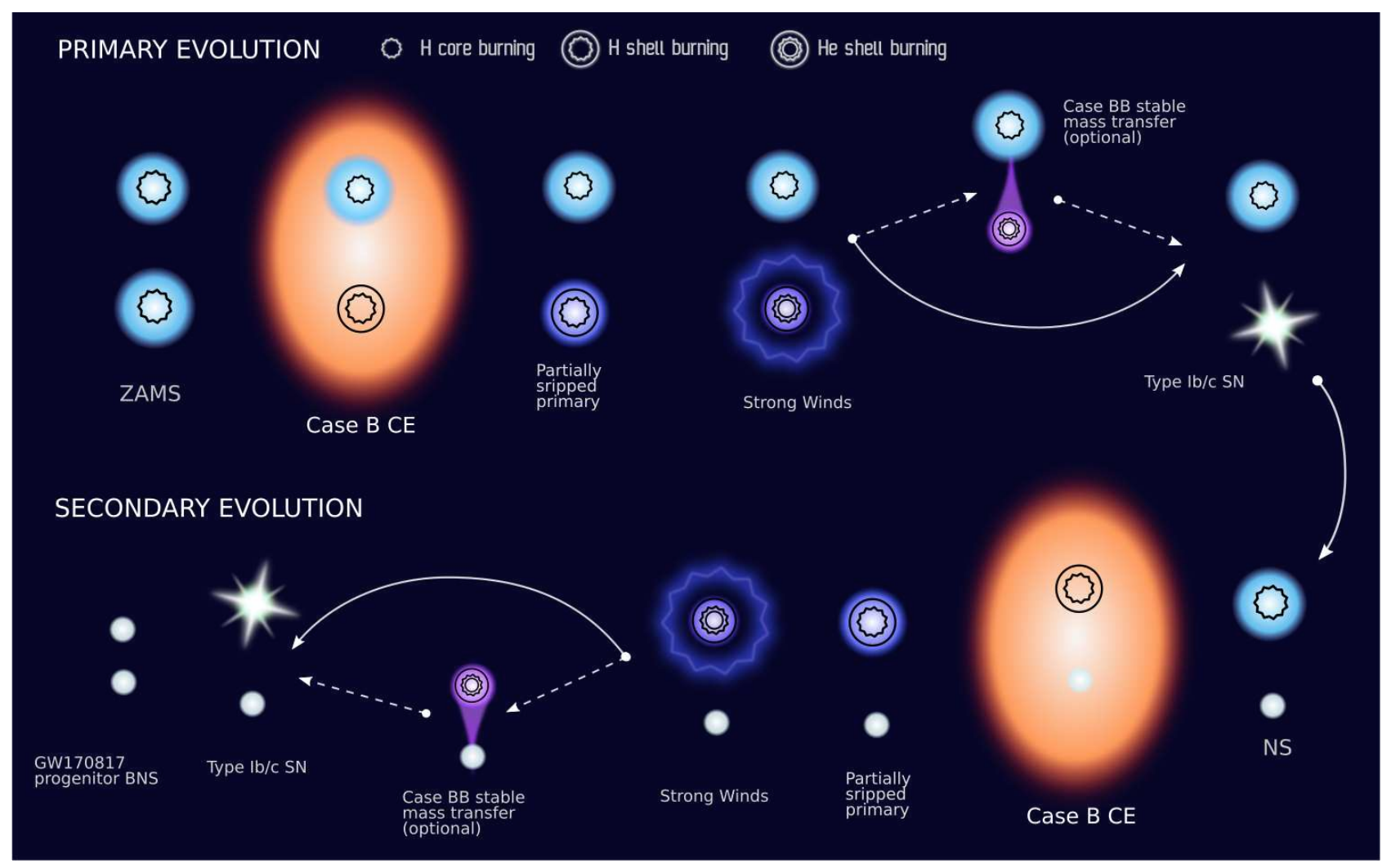

Figure 4. Most likely evolutionary pathways of GW170817 progenitors as predicted by the BPASS models.

\section{Discussion}

The age and metallicity distributions we inferred from SED fitting with our templates suggest that NGC 4993 has undergone two major episodes of star formation. The first occurred around the peak of cosmic star formation ${ }^{28}$, whilst a more recent and lower-yield star formation episode arose $\sim 1$ Gyrs ago. A similar conclusion was drawn using commonly used single star population SED templates ${ }^{29}$, but those results implied a mass contribution of $\sim 20 \%$ by the young population ${ }^{12}$, as opposed to our $<5 \%$. This is due to differences in the strength of TiO bands between 7000 and $7500 \AA$ : in BPASS their amplitude is greater at younger ages $(\sim 1 \mathrm{Gyr})$ compared to the commonly used spectra ${ }^{16}$, meaning that our templates can reproduce the features of NGC 4993 with a lower mass fraction and without requiring a very super-solar $(\mathrm{Z}=0.05)$ component previously invoked in the literature ${ }^{12}$. There is abundant evidence that NGC 4993 underwent a major merger ${ }^{12,14,30}$ and it was suggested that the young population could have resulted from that event ${ }^{12}$. However, the evolution of the shell structure of NGC 4993 is more consistent with a merger event occurring $200 \mathrm{Myrs}$ ago and very unlikely to be older than $600 \mathrm{Myrs}^{31}$. Consequently, our binary models suggest that the second episode of mass formation is unlikely to be the direct result of the major merger, and since we do not see any indication that there is a significant population of stars with ages $<500$ Myrs it would appear that it has not resulted in significant star formation.

Using the properties of the host galaxy and the chirp mass derived by LIGO/VIRGO, we find thousands of GW170817 progenitor candidates predicted by the BPASS models. A summary diagram of the evolution of the most likely progenitors of GW170817 is shown in Figure 4. We identify two families of parent systems: one with have high mass ratios $(q \gtrsim 0.55)$ and primary mass $\mathrm{M}_{1} \approx 15 \mathrm{M}_{\odot}$; another with a lower mass ratio $(0.55 \gtrsim q)$ and $\mathrm{M}_{1} \approx 25$. The genealogies we uncovered are similar to BNS production channels in the literature, yet have notable differences. Like previous studies, we find CE evolution to be ubiquitous and key to the successful formation of a BNS progenitor: In BPASS $72 \%$ of binary systems in the mass range $5-40 \mathrm{M}_{\odot}$ undergo $\mathrm{CE}^{15}$, but we find that $90 \%$ of primaries and $100 \%$ of secondary stars in our results exhibit CE. This is expected as stable mass transfer between the secondary and the primary neutron star would likely result in the formation of a black hole. We also see Case BB RLOF arise in both the evolution of the primary and the secondary star, but the frequency and efficacy of that phase is greater in the secondary evolution. Nonetheless, Case BB mass transfer is not ubiquitous and in most cases strong stellar winds remain the main source of mass-loss.

The most significant departure from the channels previously reported in the literature is the presence of a CE phase in both 
the primary and the secondary star evolution (for $90 \%$ of our star systems). BNS formation channels typically invoke a CE phase after the secondary star has left the $\mathrm{MS}^{32,33}$, and a scenario where the primary engulfs an already evolved secondary has also been proposed ${ }^{33}$, but in this case the secondary undergoes Case BB stable mass transfer with no CE. This discrepancy in the evolutionary pathways could be the result of uncertainties associated with CE evolution and the ways it is treated in different models - future constrains on CE physics may reveal that some channels are more prevalent than others or impossible altogether.

The other main source of uncertainty are supernova kicks. In our analysis so far we have used a single kick prescription for both supernova events: a uni-modal Hobbs kick distribution ${ }^{34}$. It has however been suggested that the second supernova should be an ultra-stripped supernova (USSN) with ejecta masses less than a few $0.1 \mathrm{M}_{\odot}$ and kick magnitudes an order of magnitude smaller than for a typical core collapse supernova ${ }^{32,35,36}$. Consequently, we ran simulations with an added USSN prescription (flagging any star with final mass $<2 \mathrm{M}_{\odot}$ ) and providing a randomly oriented kick of $30 \mathrm{~km} \mathrm{~s}^{-1}$, as previously seen in the literature $^{33}$ : we find no matches to our delay time and metallicity criteria. It is important to note that some of the progenitor candidates retrieved here $d o$ have second supernovae with ejecta mass $\sim 0.4 \mathrm{M}_{\odot}$, which could qualify as USSN, but they were not retrieved when applying the $30 \mathrm{~km} \mathrm{~s}^{-1}$ random kick in our follow-up simulation. The most likely cause is that the delay time resulting from that kick prescription would have prevented the model from matching our criteria (Table1). This supports the idea that USSN and low kick velocities are not a requirement for all BNS merger pathways ${ }^{37}$, although it could also indicate that further work is needed to refine the low-velocity kick distribution.

It is clear that the field is far from having reached a consensus regarding the evolutionary pathways to BNS merger. The new influx of expected gravitational wave detections from BNS mergers ${ }^{38}$ and the likely prospect of locating their optical counterparts ${ }^{39,40}$ will allow us to apply the methods presented here to a larger sample of events. Comparing progenitor searches from all available models will be key to unifying our communal understanding of massive binary star evolution and BNS mergers, but ultimately it is crucial to employ the approach presented here to study the host environment and investigate the transient genealogy using the same set of stellar evolution and population models in these analyses.

\section{Methods}

\section{SED fitting}

We used the MUSE data reduction of NGC4993 previously presented ${ }^{12}$ but performed Voronoi binning again using a different signal-to-noise ratio (SNR) target; we use the python implementation of the vorbin code ${ }^{41}$. The pixel-wise SNR provided to the algorithm is calculated by taking the average SNR between 5590 and $5680 \AA$, as this region of the spectrum is devoid of strong lines and is representative of most of the spectrum. We retrieve $>1400$ bins with SNRs $>35$ with highest SNR $\sim 65$ in the central parts of the galaxy where the pixel-wise SNR is high enough not to require voronoi binning. In order to fit BPASS synthetic spectra to our observations we use the Penalized Pixel-Fitting (ppxf) algorithm ${ }^{42}$. Pre-processing is required to make BPASS templates use-able by ppxf, and it is dependent on the observational data to be fitted. To perform this pre-processing, we developed new hoki ${ }^{43}$ features to create template SEDs and handle the ppxfoutputs.

The BPASS SEDs contain 100,000 wavelengths and are calculated for 51 ages in 13 metallicities. Because ppx ftemplates are not universal and need to be calculated for each data-set (with different dispersion and velocity scale), there is no advantage to creating templates that cover the full range of properties offered by BPASS - it would only add computational complexity during fitting. We therefore restrict the wavelength range to that of the observational data with a buffer of $+/-50 \AA$, and we constrain our templates to the 42 BPASS ages within a Hubble time: $\log ($ age $)=6.0$ to 10.1 in 0.1 increments. Although in theory all ages and metallicity can be fit simultaneously, this can result in best fits with unphysical interpretation, such as an old population with a supersolar metallicity combined with a very young component with very low metallicity. This is because the recovery of star formation histories from SED fitting is an ill-conditioned inverse problem, meaning that it suffers from severe (and unsolvable) degeneracies ${ }^{42}$.

To reduce the fitting complexity for ppxf, we restrict the range of metallicities to be included in the templates using previous estimates $^{11-13,30}$ and we implement templates constrained to $Z=\{0.01,0.014,0.020,0.030\}$. This allows us to perform simultaneous age and metallicity fits without running into a large number of unphysical solutions, although a few high metallicity templates with old ages (>10 Gyrs) are still included by the algorithm (Figure 1 - top-right panel). These are regarded as bogus and ignored in our interpretation. It is also worth noting that when fitting the population at the location of the kilonova, we do not use the voronoi tessalation; instead we integrate the flux of each pixel contained between a 1.5 " and a 2 " annulus ${ }^{12}$ centered on AT $2017 \mathrm{gfo}$, and the spectrum of the transient (integrated within a 0.5 " annulus) is removed to isolate the stellar component. In Table 2 we provide the values shown graphically in Figure 2.

\section{Model Search and Computational Methods}

Throughout this work we use BPASSv2.2.1 ${ }^{16}$ with the fiducial initial mass function ${ }^{44}$ with maximum mass $=300 \mathrm{M}_{\odot}$. In order to retrieve the full evolutionary pathway of a predicted BNS we must search our stellar library, where unique models are defined 
Table 2. Results from fitting the stellar population in an annulus around AT2017gfo.

\begin{tabular}{ccccc}
\hline Metallicity & $\log$ (age.years) & ppxf weight & Light Fraction & Mass Fraction \\
\hline 0.02 & 8.9 & 0.0394 & 0.0485 & 0.0122 \\
0.03 & 9.0 & 0.0783 & 0.0965 & 0.0363 \\
0.01 & 9.5 & 0.2766 & 0.3408 & 0.2471 \\
0.01 & 9.7 & 0.3114 & 0.3837 & 0.4229 \\
0.01 & 9.9 & 0.1059 & 0.1305 & 0.2816 \\
\hline
\end{tabular}

by a combination of initial primary mass, secondary mass and period. For computational reasons the detailed evolution of the primary and secondary star are calculated separately and located in separate files - we therefore must pair corresponding primary star and secondary star evolutionary histories. The right pairing is dependent on the random kick imparted by the first supernova, as different kicks will result in a different period and separation or in the disruption of the system altogether. We must also consider kicks imparted by the second supernova as they will have a direct impact on the birth period and eccentricity of the BNS, impacting their delay time and potentially leading to the disruption of the binary. In these simulations we randomly sampled a uni-modal Maxwellian distribution with $\sigma=265 \mathrm{~km} \mathrm{~s}^{-134}$ to determine the amplitude, and a uniform distribution for the orientation. We performed 10,000 iterations for each supernova, resulting in as many as $10^{8}$ kicks per system.

Once the BNS systems have been created their merger time is computed with the Mandel approximation ${ }^{45}$. Since our mass ratios are well within the boundary conditions we do not expect deviations larger than 3 percent from the typical integration, and the computational efficiency of this solution allows more kicks to be sampled. As mentioned in the main text, our numerical simulations record systems with BNS systems with chirp-mass within $0.1 \mathrm{M}_{\odot}$ of the chir-pmass of GW170817. It is important to note however that the quoted chirp-mass is the gravitational mass, whereas BPASS predictions provide baryonic masses. Consequently we scale the reported value of 1.186 using $M_{b}=M_{g}+0.080 M_{g}^{2}$, where $\mathrm{M}_{b}$ is the baryonic mass and $\mathrm{M}_{g}$ is the gravitational mass ${ }^{46}$.

The results of these simulations are not currently public, unlike the core BPASS outputs and stellar models. Additionally, the hok i features used to create SED templates, make delay time cuts, and recover the genealogy of our progenitor candidates, have not yet been released on the public repository. We are happy to share our results and discuss our pipelines on request.

\section{References}

1. Abbott, B. P. et al. Multi-messenger Observations of a Binary Neutron Star Merger. The Astrophys. J. 848, L12, DOI: 10.3847/2041-8213/aa91c9 (2017).

2. Coulter, D. A. et al. Swope Supernova Survey 2017a (SSS17a), the optical counterpart to a gravitational wave source. Science 358, 1556-1558, DOI: 10.1126/science.aap9811 (2017).

3. Evans, P. A. et al. Swift and NuSTAR observations of GW170817: Detection of a blue kilonova. Science 358, 1565-1570, DOI: 10.1126/science.aap9580 (2017).

4. Villar, V. A. et al. The Combined Ultraviolet, Optical, and Near-Infrared Light Curves of the Kilonova Associated with the Binary Neutron Star Merger GW170817: Unified Data Set, Analytic Models, and Physical Implications. ApJ 851, L21, DOI: $10.3847 / 2041-8213 / \mathrm{aa} 9 \mathrm{c} 84$ (2017). 1710.11576.

5. Nicholl, M. et al. The Electromagnetic Counterpart of the Binary Neutron Star Merger LIGO/Virgo GW170817. III. Optical and UV Spectra of a Blue Kilonova from Fast Polar Ejecta. The Astrophys. J. 848, L18, DOI: 10.3847/2041-8213/aa9029 (2017).

6. Chornock, R. et al. The Electromagnetic Counterpart of the Binary Neutron Star Merger LIGO/Virgo GW170817. IV. Detection of Near-infrared Signatures of r-process Nucleosynthesis with Gemini-South. The Astrophys. J. 848, L19, DOI: 10.3847/2041-8213/aa905c (2017).

7. Smartt, S. J. et al. A kilonova as the electromagnetic counterpart to a gravitational-wave source. Nature 551, 75-79, DOI: 10.1038/nature24303 (2017).

8. Tanvir, N. R. et al. The Emergence of a Lanthanide-rich Kilonova Following the Merger of Two Neutron Stars. The Astrophys. J. 848, L27, DOI: 10.3847/2041-8213/aa90b6 (2017).

9. Hjorth, J. et al. The Distance to NGC 4993: The Host Galaxy of the Gravitational-wave Event GW170817. The Astrophys. J. 848, L31, DOI: 10.3847/2041-8213/aa9110 (2017).

10. Cantiello, M. et al. A Precise Distance to the Host Galaxy of the Binary Neutron Star Merger GW170817 Using Surface Brightness Fluctuations. The Astrophys. J. 854, L31, DOI: 10.3847/2041-8213/aaad64 (2018). 
11. Blanchard, P. K. et al. The Electromagnetic Counterpart of the Binary Neutron Star Merger LIGO/Virgo GW170817. VII. Properties of the Host Galaxy and Constraints on the Merger Timescale. The Astrophys. J. 848, L22, DOI: 10.3847/ 2041-8213/aa9055 (2017).

12. Levan, A. J. et al. The Environment of the Binary Neutron Star Merger GW170817. The Astrophys. J. 848, L28, DOI: 10.3847/2041-8213/aa905f (2017).

13. Pan, Y. C. et al. The Old Host-galaxy Environment of SSS17a, the First Electromagnetic Counterpart to a Gravitational-wave Source. The Astrophys. J. 848, L30, DOI: 10.3847/2041-8213/aa9116 (2017).

14. Im, M. et al. Distance and Properties of NGC 4993 as the Host Galaxy of the Gravitational-wave Source GW170817. The Astrophys. J. 849, L16, DOI: 10.3847/2041-8213/aa9367 (2017).

15. Eldridge, J. J. et al. Binary Population and Spectral Synthesis Version 2.1: Construction, Observational Verification, and New Results. Publ. Astron. Soc. Aust. 34, e058, DOI: 10.1017/pasa.2017.51 (2017).

16. Stanway, E. R. \& Eldridge, J. J. Re-evaluating old stellar populations. Mon. Notices Royal Astron. Soc. 479, 75-93, DOI: 10.1093/mnras/sty1353 (2018).

17. Eldridge, J. J., Fraser, M., Smartt, S. J., Maund, J. R. \& Crockett, R. M. The death of massive stars - II. Observational constraints on the progenitors of Type Ibc supernovae. Mon. Notices Royal Astron. Soc. 436, 774-795, DOI: 10.1093/ mnras/stt1612 (2013).

18. Steidel, C. C. et al. Reconciling the Stellar and Nebular Spectra of High-redshift Galaxies. 826, 159, DOI: 10.3847/ 0004-637X/826/2/159 (2016). 1605.07186.

19. Bestenlehner, J. M. et al. The R136 star cluster dissected with Hubble Space Telescope/STIS - II. Physical properties of the most massive stars in R136. 499, 1918-1936, DOI: 10.1093/mnras/staa2801 (2020). 2009.05136.

20. Stevance, H. F. \& Eldridge, J. J. Binary pathways to SLSNe-I: SN 2017gci. 504, L51-L55, DOI: 10.1093/mnrasl/slab039 (2021). 2104.03365.

21. Runco, J. N. et al. The MOSDEF survey: a comprehensive analysis of the rest-optical emission-line properties of $\mathrm{z} \sim 2.3$ star-forming galaxies. 502, 2600-2614, DOI: 10.1093/mnras/stab119 (2021). 2008.04924.

22. Stevance, H. F., Parsons, S. G. \& Eldridge, J. J. NGC 1850 BH1: To be or not to be a black hole? arXiv e-prints arXiv:2112.00015 (2021). 2112.00015.

23. Briel, M. M., Eldridge, J. J., Stanway, E. R., Stevance, H. F. \& Chrimes, A. A. Estimating Transient Rates from Cosmological Simulations and BPASS. arXiv e-prints arXiv:2111.08124 (2021). 2111.08124.

24. Abbott, B. P. et al. On the Progenitor of Binary Neutron Star Merger GW170817. The Astrophys. J. 850, L40, DOI: 10.3847/2041-8213/aa93fc (2017).

25. Eldridge, J. J., Stanway, E. R. \& Tang, P. N. A consistent estimate for gravitational wave and electromagnetic transient rates. Mon. Notices Royal Astron. Soc. 482, 870-880, DOI: 10.1093/mnras/sty2714 (2019).

26. Hachinger, S. et al. How much $\mathrm{H}$ and He is 'hidden' in $\mathrm{SNe} \mathrm{Ib} / \mathrm{c}$ ? - I. Low-mass objects. 422, 70-88, DOI: 10.1111/j. 1365-2966.2012.20464.x (2012). 1201.1506.

27. Gilkis, A. \& Arcavi, I. How much hydrogen is in Type Ib and IIb supernova progenitors? arXiv:2111.04432 [astro-ph] (2021). 2111.04432.

28. Madau, P. \& Dickinson, M. Cosmic Star-Formation History. 76.

29. Bruzual, G. \& Charlot, S. Stellar population synthesis at the resolution of 2003. Mon. Notices Royal Astron. Soc. 344, 1000-1028, DOI: 10.1046/j.1365-8711.2003.06897.x (2003).

30. Palmese, A. et al. Evidence for Dynamically Driven Formation of the GW170817 Neutron Star Binary in NGC 4993. The Astrophys. J. 849, L34, DOI: 10.3847/2041-8213/aa9660 (2017).

31. Ebrová, I., Bílek, M., Yıldız, M. K. \& Eliášek, J. NGC 4993, the shell galaxy host of GW170817: Constraints on the recent galactic merger. Astron. \&amp; Astrophys. Vol. 634, id.A73, <NUMPAGES $>11</ N U M P A G E S>$ pp. 634, A73, DOI: 10.1051/0004-6361/201935219 (2020).

32. Tauris, T. M. et al. Formation of Double Neutron Star Systems. The Astrophys. J. 846, 170, DOI: 10.3847/1538-4357/aa7e89 (2017).

33. Vigna-Gómez, A. et al. On the formation history of Galactic double neutron stars. Mon. Notices Royal Astron. Soc. 481, 4009-4029, DOI: 10.1093/mnras/sty2463 (2018). 1805.07974. 
34. Hobbs, G., Lorimer, D. R., Lyne, A. G. \& Kramer, M. A statistical study of 233 pulsar proper motions. Mon. Notices Royal Astron. Soc. 360, 974-992, DOI: 10.1111/j.1365-2966.2005.09087.x (2005).

35. Tauris, T. M. et al. ULTRA-STRIPPED TYPE Ic SUPERNOVAE FROM CLOSE BINARY EVOLUTION. ApJ 778, L23, DOI: 10.1088/2041-8205/778/2/L23 (2013).

36. Tauris, T. M., Langer, N. \& Podsiadlowski, P. Ultra-stripped supernovae: Progenitors and fate. Mon. Notices Royal Astron. Soc. 451, 2123-2144, DOI: 10.1093/mnras/stv990 (2015).

37. Chu, Q., Yu, S. \& Lu, Y. Formation and Evolution of Binary Neutron Stars: Mergers and Their Host Galaxies. arXiv:2110.04687 [astro-ph] (2021). 2110.04687.

38. Abbott, B. P. et al. Prospects for observing and localizing gravitational-wave transients with advanced ligo, advanced virgo and kagra. Living Rev. Relativ. 23, DOI: 10.1007/s41114-020-00026-9 (2020).

39. Nguyen, C. Status of the advanced virgo gravitational-wave detector (2021). 2105.09247.

40. Dyer, M. J. et al. Developing the goto telescope control system. Softw. Cyberinfrastructure for Astron. VI DOI: 10.1117/12.2561506 (2020).

41. Cappellari, M. \& Copin, Y. Adaptive spatial binning of integral-field spectroscopic data using Voronoi tessellations. Mon. Notices Royal Astron. Soc. 342, 345-354, DOI: 10.1046/j.1365-8711.2003.06541.x (2003).

42. Cappellari, M. Improving the full spectrum fitting method: Accurate convolution with Gauss-Hermite functions. Mon. Notices Royal Astron. Soc. 466, 798-811, DOI: 10.1093/mnras/stw3020 (2017).

43. Stevance, H., Eldridge, J. \& Stanway, E. Hoki: Making BPASS accessible through Python. The J. Open Source Softw. 5, 1987, DOI: 10.21105/joss.01987 (2020).

44. Kroupa, P. On the variation of the initial mass function. Mon. Notices Royal Astron. Soc. 322, 231-246, DOI: 10.1046/j. 1365-8711.2001.04022.x (2001).

45. Mandel, I. \& Broekgaarden, F. S. Rates of Compact Object Coalescences. arXiv:2107.14239 [astro-ph] (2021). 2107.14239.

46. Gao, H. et al. Relation between gravitational mass and baryonic mass for non-rotating and rapidly rotating neutron stars. Front. Phys. 15, 24603, DOI: 10.1007/s11467-019-0945-9 (2020). 1905.03784.

\section{Acknowledgements (not compulsory)}

HFS and JJE acknowledge the support of the Marsden Fund Council managed through Royal Society Te Aparangi. HFS is thankful to Lorenza Della Bruna, Angela Adamo and Chris Usher for private communications regarding the voronoi binning and ppxf algorithms. HFS is grateful to Stephen Smartt for his comments on the final draft of the manuscript. ERS acknowledges funding from the UK Science and Technology Facilities Council (STFC) through Consolidated Grant ST/T000406/1. AJL has received funding from the European Research Council (ERC) under the European Union's Horizon 2020 research and innovation programme (Grant agreement No.725246).

\section{Author contributions statement}

H.F.S. is the lead developer of hoki, created the pipelines to make BPASS SED templates, refactored the TUI codebase, performed the SED fitting, and lead the writing of this manuscript. J.J.E. conceived the project, is one of the lead developers of BPASS, wrote TUI, and advised throughout the analysis. E.S. is one of the lead developers of BPASS and commented on early and later drafts of the manuscript. J.L. provided relevant expertise on the SED fitting procedure and analysis of NGC4993 as well as the data reduction used for this paper. A.F.M. advised on the handling of MUSE data and SED fitting. A.J.L. led initial acquisition of the data, provided text and advised on the manuscript.

\section{Additional information}

Competing interests: There are no competing interests to report. 